\title{
Experimental Study of Noise Emitted by Circular Cylinders with Large Roughness
}

\author{
Antoni Alomar, David Angland $†$ and Xin Zhang ${ }^{\ddagger}$ \\ University of Southampton, Southampton, Hampshire, SO17 1BJ, UK
}

\begin{abstract}
A set of experiments of rough circular cylinder flow as well as rough wall turbulent boundary layer (TBL) flow were performed with the goal of studying the effect of large roughness on the aerodynamic noise emitted by single bluff bodies. Roughness consisted of uniformly distributed hemispherical and cylindrical elements with normalised heights of 0.031 and $\mathbf{0 . 0 3 5}$, respectively, as well as cable helicoidally wrapped with normalised heights of 0.031 and 0.047 . The Reynolds numbers in the circular cylinder tests ranged between $1.6 \times 10^{5}$ and $3.2 \times 10^{5}$. A broadband spectral peak has been identified mainly in the hemispherical roughness configurations, centred at frequencies well predicted by Howe's roughness noise prediction model that was extended to the case of a circular cylinder. The peak level was overpredicted and for higher frequencies the difference increased. The helicoidal cable configurations didn't generate significant roughness noise and, furthermore, its far field noise spectra have been found to collapse with the smooth cylinder spectra for Strouhal numbers greater than 0.7. The observed effect of roughness at lower frequencies was to increase the vortex shedding peak level and to reduce the peak Strouhal number, which is in accordance with the observations of previous studies.
\end{abstract}

\section{Nomenclature}

$c_{o} \quad$ Sound speed

$c_{f} \quad$ Friction coefficient

$D \quad$ Cylinder diameter

$D_{f} \quad$ Directivity function

$h \quad$ Mean roughness element height

$f \quad$ Frequency

$f_{r n} \quad$ Roughness noise peak frequency

$f_{v s} \quad$ Vortex shedding fundamental frequency

$G$ Green function

k Wavenumber components

$k_{o} \quad$ Acoustic wavenumber magnitude

$K_{R}$ Proportionality constant in Smol'yakov prediction model

$L \quad$ Circular cylinder length

$L_{R} \quad$ Streamwise length of the rough surface

$L_{R}^{\prime} \quad$ Spanwise length of the rough surface

$M \quad$ Mach number

$N \quad$ Number of roughness elements per unit area

$p \quad$ Acoustic pressure

$P \quad$ Power spectral density

$p_{\text {ref }}$ Reference pressure

$p_{r m s}$ Root-mean-square acoustic pressure

$P_{R} \quad$ Rough wall acoustic pressure spectrum

${ }^{*} \mathrm{PhD}$ Student, Faculty of Engineering and the Environment, Student Member AIAA.

${ }^{\dagger}$ Research Fellow, Faculty of Engineering and the Environment, Member AIAA.

$\ddagger$ Airbus Professor of Aircraft Engineering, Faculty of Engineering and the Environment, Associate Fellow AIAA. 
$P_{S} \quad$ Smooth-wall pressure wavenumber-frequency spectrum

$r \quad$ Distance from cylinder axis to observer

Re Reynolds number based on cylinder diameter

$S \quad$ Rough area

St Strouhal number based on cylinder diameter

$S t_{w}$ Roughness element Strouhal number in Smol'yakov prediction model

$U_{o} \quad$ Freestream velocity

$U_{o}(\phi)$ Potential flow velocity

$U_{c} \quad$ Convection velocity

$u_{\tau} \quad$ Friction velocity

$\mathrm{x}$ Observer location

y Source location

Greek symbols

$\delta \quad$ Boundary layer thickness

$\delta^{*} \quad$ Boundary layer displacement thickness

$\zeta \quad$ Helicoidal angle

$\theta, \phi \quad$ Observation angles

$\mu_{H} \quad$ Roughness density factor

$\rho_{o} \quad$ Ambient density

$\sigma \quad$ Surface roughness density

$\Phi \quad$ Point pressure spectrum

$\hat{\Phi} \quad$ Surface pressure wavenumber-frequency spectrum

$\phi \quad$ Azimuthal angle measured from the upstream stagnation line

$\Phi_{S} \quad$ Function in Smol'yakov prediction model

$\Psi \quad$ Function related with the surface roughness density

$\omega \quad$ Angular frequency

Subscript

i Variable number

Superscript

$+\quad$ Variable normalised with inner variables

\section{Introduction}

There is an increasing interest in understanding and predicting airframe noise radiated by civil aircraft during approach-to-landing. Current practical landing gear noise prediction models are component-based, so that the various components are divided in groups according to the dominant frequency range in which they radiate noise. ${ }^{1}$ Since the far field noise spectra are approximately Strouhal-based the emitted frequency is assumed to be directly related to their size; the large elements are responsible for the low frequency region of the spectra, and the small components for the high frequency region. The large elements (wheels, bogie beam, main strut) are modelled using either empirically or computationally determined far field noise spectra of these large elements in isolation and assuming a smooth surface. The high complexity of the small components means they are either neglected ${ }^{2,3}$ or modelled empirically. In Guo ${ }^{1}$ the small components were described using a 'complexity factor', which is an empirical function of global geometrical parameters of the landing gear and the aircraft size. The predictive power of this approach is limited to the specific gear configurations used to determine the model constants, and is not capable of predicting how changes in the size and location of the small elements affect the radiated noise. It was shown that for the predictions to fit the measured far field noise data the small components, which in the component-based approach contribute mainly in the high frequency range, have a dominant role for frequencies typically higher than approximately $1 \mathrm{kHz}$ (for the main gear of a typical medium range aircraft). ${ }^{1}$ Therefore, if the predictive accuracy for high frequencies is to be increased, the modelling of the small elements needs improvement.

The small components typically have a wide range of shapes and surface distributions on the larger elements. They are characterized by the fact that their size is much smaller than the size of the large elements. On the upstream face of the large components and in absence of large element interactions, a TBL 
will grow until it separates, causing some of the small components to be immersed in it. This situation is known to enhance the scattering of turbulent sound sources in the same way as any vortical flow interacting with a compact body, ${ }^{4}$ and the phenomenon is described by roughness noise. There exist several models in the literature able to calculate the radiated noise by rough flat walls immersed in a TBL, in terms of the roughness geometry and the Reynolds stresses of the TBL.

The need for improving the predictive accuracy in the high frequency range necessitates an improvement of the modelling of small components. In this study we have attempted an approach to the problem of analytically modelling the noise radiated by the small components, as well as a study on their effect in lower frequencies. The existing theory of roughness noise has been used and extrapolated to the case of single bluff bodies. As a first approach a set of simple configurations have been chosen, with uniform roughness size and roughness shape distributed over the bluff body surface.

In section II the flat wall roughness noise prediction models appearing in the literature are briefly summarised. In section III the experimental methods are described, together with the roughness configurations. In section IV the results are presented and discussed, and finally section V contains the conclusions.

\section{Background}

There exist several models of relevance to the present problem. The flat wall roughness noise model developed by Howe ${ }^{5}$ was implemented by Liu and Dowling. ${ }^{6,7}$ They included an exact numerical integration over the wavenumber space in the calculation of the far field noise instead of an asymptotic expansion around the convective ridge used by Howe. They also compared the predictions with experimental measurements of the radiated noise by a rough flat wall, where the roughness elements were hemispheres, as the model assumes. The size of the bosses was large compared with the TBL thickness $(h / \delta \approx 0.5$ in the leading row of elements), and the flow was in the fully-rough regime $\left(h^{+}>300\right)$. The model predicted the peak level well and the peak frequency for two roughness sizes and surface densities. It uses a Green function tailored to the rough wall geometry. The roughness elements consist of acoustically compact and independent hemispheres, and the Lighthill analogy is used to calculate the far field noise. For a small surface element of area $S$, the radiated sound can be expressed as:

$$
P_{R}(\mathbf{x}, \omega)=\frac{S \sigma \mu_{H}^{2}}{4|\mathbf{x}|^{2}} \frac{h^{4}}{\delta^{* 4}} \frac{U_{c}^{2}}{c^{2}} \Phi(\omega) D_{f}(\theta, \phi),
$$

where $\delta^{*}$ is the TBL displacement thickness, and the function $D_{f}(\theta, \phi)$ contains the directivity information of the emitted sound:

$$
\begin{gathered}
D_{f}(\theta, \phi)=Z_{1} \cos ^{2} \theta+Z_{2} \sin ^{2} \theta \sin ^{2} \phi+2 Z_{3} \cos \theta \sin \theta \sin \phi, \\
Z_{1}=\int_{0}^{\infty} \int_{0}^{2 \pi} \Gamma \cos ^{2} \eta d\left(|\mathbf{k}| \delta^{*}\right) d \eta \\
Z_{2}=\int_{0}^{\infty} \int_{0}^{2 \pi} \Gamma \sin ^{2} \eta d\left(|\mathbf{k}| \delta^{*}\right) d \eta \\
Z_{3}=\int_{0}^{\infty} \int_{0}^{2 \pi} \Gamma \sin \eta \cos \eta d\left(|\mathbf{k}| \delta^{*}\right) d \eta, \\
\Gamma=|\gamma(\mathbf{k})|^{2} \delta^{* 2} \hat{\Phi}(\mathbf{k}, \omega) \Psi(|\mathbf{k}|) e^{-2|\mathbf{k}| h}\left(|\mathbf{k}| \delta^{*}\right) .
\end{gathered}
$$

The $|\mathbf{k}| \delta^{*}$-integral upper limit must be specified. Liu and Dowling argue that the weighting factor $e^{-2|\mathbf{k}| h}$ assures the convergence of the integral and that $|\mathbf{k}| \delta^{*}<25 \delta^{*} / h$ is large enough for practical purposes. The point pressure spectrum $\Phi(\omega)$ used is the one proposed by Ahn, ${ }^{8}$ which better fits the experimental data in the frequency range of interest than other models. Also Corcos model ${ }^{9}$ of the surface pressure wavenumberfrequency spectrum has been used.

The model predicts the far field noise spectrum scales with $U_{o}^{6}$, i.e. the sound sources are equivalent to a distribution of surface dipoles. It also predicts that the peak frequency for $\sigma \approx 0.5$ is located in $\omega h / u_{\tau} \approx 18$. 
The friction velocity changes (diminishes) along the TBL, but the change is small for moderate lengths. As a first approximation it is $u_{\tau} \approx 0.07 U_{o}$, and therefore $f h / U_{o} \approx 0.2(\omega=2 \pi f)$.

Another model was developed by Smol'yakov, ${ }^{11}$ which assumes that the roughness elements protrude into the outer region of the TBL and each roughness element acts as an independent, acoustically compact bluff body. This is more relevant to the case of the small details in a landing gear, when $h / \delta$ is large. Smol'yakov argues that the flow mechanism responsible for noise emission is Strouhal-based but with an unknown constant value of the Strouhal number $S t_{w}$. In this approach, each roughness element emits tonal noise at a frequency $\omega=S t_{w} U_{r} / h$, where $U_{r}$ is the effective velocity impinging on the element, and $h$ is the roughness element size. $U_{r}, h$ and $\omega$ are treated as statistical distributions. The mean value of $U_{r}$ is $8.5 u_{\tau} \approx 0.5 U_{o}$, according to Schlichting and Gersten. ${ }^{12}$ Smol'yakov imposed the probability density function of the noise frequency to be proportional to the noise spectrum $P_{R}(\mathbf{x}, \omega)$, and he reached:

$$
\begin{gathered}
P_{R}(\mathbf{x}, \omega)=K_{R} \frac{S}{|\mathbf{x}|^{2}} c_{f}^{5 / 2} h \rho_{o}^{2} U_{o}^{2} M^{2} \Phi_{S}\left(\frac{\omega h}{U_{o \sqrt{c_{f}}} 6 S t_{w}}\right) \\
\Phi_{S}(\xi)=\int_{0}^{\infty} z^{6} e^{-\xi^{2} z^{2}+(8.5 \sqrt{2} \xi-1) z} d z
\end{gathered}
$$

where $K_{R}$ is a proportionality constant. The parameters $K_{R}$ and $S t_{w}$ must be determined by measurements. It should be noted that this model does not include directivity information.

The effect of surface roughness on circular cylinder flow has been widely studied experimentally in the past. However no studies have been performed regarding the noise radiation of highly rough cylinders. Achenbach and Heinecke ${ }^{13}$ measured coherent vortex shedding in the supercitical and postcritical regimes even for the largest roughness $(h / D=0.03)$, which had pyramidal shape. The measured Strouhal number was $S t \approx 0.22$ for the largest roughness. Achenbach ${ }^{14}$ measured the mean drag coefficient of cylinders covered with glued spheres with $h / D=0.017$ over all the Reynolds number range. The postcritical values of mean drag coefficient obtained were close to the subcritical regime value, and increased with increasing roughness size. The results were interpreted by Guven et al. ${ }^{15}$ through the effect that roughness has on the TBL's present on the upstream face of the cylinder. The presence of coherent vortex shedding requires the separation lines to be straight along the span, i.e. the surface geometry should allow the TBL's to be straight. ${ }^{17}$ If the roughness elements are large enough with respect to the cylinder diameter the TBL's will potentially not be well developed before separation which will prevent coherent vortex shedding. The presence of a helicoidal cable wrapped around circular cylinders has been shown to affect vortex shedding by breaking down the spanwise coherence. However the effectiveness in the vortex shedding suppression is very dependent on the cable density and helicoidal angle. ${ }^{19}$ Cylinders with circumferential grooves, equivalent to a dense helicoidal cable, appeared to have the same effect as conventional 3-dimensional roughness. ${ }^{18}$

\section{Experimental Arrangement}

\section{A. Facilities}

Far field acoustic measurements and on-surface pressure measurements were performed in an anechoic chamber equipped with an open jet facility at the University of Southampton (see figure 1). The cross-section of the nozzle is rectangular and has dimensions of $500 \mathrm{~mm}$ (height) $\times 350 \mathrm{~mm}$ (width). The maximum speed of the facility is $40 \mathrm{~m} / \mathrm{s}$, and the incoming turbulence level is $0.2 \%$ outside of the shear layers. ${ }^{20}$ Phased microphone array measurements, PIV and Pitot tube measurements were conducted in a low speed wind tunnel at the University of Southampton. The tunnel is an open-loop, closed-section configuration, with a rectangular test section of dimensions $600 \mathrm{~mm}$ (height) $\times 900 \mathrm{~mm}$ (width). The maximum speed is $27 \mathrm{~m} / \mathrm{s}$ (measured at the test section entrance). The incoming turbulence intensity is approximately $0.2 \%{ }^{21}$

\section{B. Instrumentation}

The far field sound level was measured in the anechoic facility using a set of microphones fixed to a metallic arc. They were located in the positions listed in Table 1 . In both cases all microphones were located in the midspan plane of the flat plate and cylinder. The observer distance $r$ and angle $\theta$ are defined in figure 4 .

The microphones are Behringer, omni-directional electret condenser ECM8000, with a frequency range of $15 \mathrm{~Hz}-20 \mathrm{kHz}$. The signals were amplified using a DIGIMAX FS by Presonus, and acquired using 


\begin{tabular}{cccccc} 
Circular cylinder & $r(\mathrm{~m})$ & $\theta\left(^{\circ}\right)$ & Flat plate & $r(\mathrm{~m})$ & $\theta\left(^{\circ}\right)$ \\
\hline M1 & 1.69 & 86 & M1 & 1.47 & 131 \\
M2 & 1.05 & 67 & M2 & 1.27 & 121 \\
M3 & 1.66 & 45 & M3 & 1.00 & 90 \\
M4 & 1.85 & 39 & M4 & 1.36 & 70 \\
M5 & 2.22 & 28 & M5 & 1.40 & 62 \\
& & & M6 & 1.71 & 48 \\
& & & M7 & 2.54 & 35
\end{tabular}

Table 1. Location of the far field microphones for circular cylinder and flat plate tests

National Instruments PXI-4472 data acquisition cards. The calibration of the microphones was performed by comparison with a $1 / 2$ " BK 4961 standard microphone. The power spectra of the pressure signals was determined using the equation:

$$
P(\omega)=\frac{2|\mathrm{FFT}(p(t))|^{2}}{n \cdot F_{s}},
$$

where $p(t)$ is the pressure signal measured, $n=8192$ is the number of samples per block, $F s=48 \mathrm{kHz}$ is the sampling frequency, and FFT is the abbreviation for Fast Fourier Transform. The frequency resolution is therefore $5.9 \mathrm{~Hz}$, and the spectra have been averaged over 120 blocks. No windowing has been applied in the calculation of the Fast Fourier Transform. The spectra have been plotted as a power spectral density as follows:

$$
\operatorname{PSD}(\omega)=10 \log _{10} \frac{P(\omega)}{p_{\text {ref }}^{2}}
$$

where $p_{\text {ref }}=2 \times 10^{-5} \mathrm{~Pa}$.

In the anechoic tests, on-surface pressure signals were measured using ECM-10B, electret condenser microphones flush mounted to the wall. The microphones have an approximately omni-directional response and a frequency range of $50 \mathrm{~Hz}-13 \mathrm{kHz}$. Their sensitivity is $-60 \pm 3 \mathrm{~dB}$ and their dimensions are $6 \mathrm{~mm}$ in diameter and $5.2 \mathrm{~mm}$ in thickness. The signals were amplified using an in-house built amplifier with adjustable gain, and acquired with National Instruments PXI-4472 data acquisition cards.

For the source location in the low speed tunnel a phased microphone array was employed, which is composed of 49 Panasonic WM-61 A electret microphones, with a frequency range of $15 \mathrm{~Hz}-20 \mathrm{kHz}$. The array was flush mounted on a square plate of dimensions $200 \mathrm{~mm} \times 200 \mathrm{~mm}$, which was mounted on the side wall of the low speed wind tunnel. The microphones were located at the intersections of a multi-arm logarithmic spiral with a set of concentric circles. The beamforming algorithm is of delay-and-sum type, and is implemented in an in-house code. ${ }^{22}$ It includes an algorithm to partly eliminate the effect of the TBL present on the microphone array.

A PIV system (Quantel Twins BSL 200) was used in the low speed wind tunnel. The pulse gap between image pairs was set at $18 \mu \mathrm{s}$. The data was processed using a recursive Nyquist grid method, from 64 to 32 pixel spot dimensions. A total of 500 to 700 image pairs were averaged to obtain the mean and root-mean-square (RMS) velocity fields.

The measurement setup wasn't changed during the tests of the various roughness configurations, and therefore the systematic error was constant for all the cases. The repeatability of all the measurements was checked, both short and long term, as well as a direct comparison between the low speed wind tunnel and the anechoic facility.

Short term repeatability tests were performed for all configurations at the higher speed. Long term repeatability tests of the smooth cylinder far field measurements show that the difference in level of the $1 / 3$-octave band spectra is smaller than approximately $0.9 \mathrm{~dB}$ at the higher speed, in the frequency range of interest. In the case of the flat plate, the long term repeatability tests showed a difference in level smaller than $0.7 \mathrm{~dB}$ at the higher speed, in the frequency range of interest. 
To check the repeatability between the low speed wind tunnel and the anechoic facility, an on-surface microphone was placed at the downstream stagnation line, and the spectra in both cases was compared at the highest flow speed. The difference in value between them was less than approximately $1 \mathrm{~dB}$ in the frequency range of interest.

The background noise levels corresponding to the empty test section configuration were measured as well, and compared with the smooth cylinder noise levels. In the case of the cylinder the signal-to-noise ratio of the smooth case was too low at the smallest speed, so this case was discarded to calculate the velocity scaling. In the case of the flat plate the difference in level between the rough and the smooth plates was high enough for all speeds and roughness configurations.

\section{Wind tunnel setup and roughness configurations}

In the acoustic measurements the circular cylinder was mounted between two endplates fixed to the nozzle. The aspect ratio of the cylinder was 2.8 and the open-jet blockage was $25 \%$. The flat plate was fixed in the centre of the nozzle. The leading edge was streamlined. In the aerodynamic experiments that took place in the low speed wind tunnel the cylinder was fixed between two endplates with an aspect ratio of 3.9 and a blockage ratio of $15 \%$. The flat plate used had a width equal to the test section width. It was supported by two streamlined struts and fixed at a height of $10 \mathrm{~cm}$ from the wind tunnel floor, outside of the influence of the boundary layer growing along the test section. The plate was equipped with a trailing edge flap and static pressure taps to ensure a zero pressure gradient.

The baseline configurations are a smooth circular cylinder and a smooth flat plate. Two roughness strips at $\pm 50^{\circ}$ measured from the upstream stagnation line were attached on the circular cylinder, which consisted of carborundum particles of average size $2.7 \cdot 10^{-4} \mathrm{~m}$ (Grit 60 ) glued to a double-sided adhesive tape. The goal of the strips was to obtain turbulent separation, and therefore supercritical-like flow. A roughness strip was also attached to the flat plate in both the anechoic and the low speed wind tunnel tests. It was located immediately downstream of the leading edge, causing the TBL to be fully developed upstream of the rough surface.

The distributed roughness configurations consist of densely packed hemispheres and circular cylinders (see figure 2) with heights $h / D=0.031$ and 0.035 , corresponding to surface densities of 0.42 and 0.53 . They covered all the span of both the flat plate and the circular cylinder. In the flat plate configuration two different distances from the leading edge to the first row of roughness elements were considered: 145 $\mathrm{mm}$ and $320 \mathrm{~mm}$. In the first case the TBL thickness exceeds the roughness height over the entire rough surface, as the prediction models assume. In the second case the TBL thickness immediately upstream of the rough surface was smaller than the roughness height. Finally a set of configurations with cable attached helicoidally to the cylinder were tested. The cable diameters were $4 \mathrm{~mm}$ and $6 \mathrm{~mm}$, and for each of them three helicoidal angles $\zeta$ was tested: $79^{\circ}, 85^{\circ}$ and $88^{\circ}$ (see figure 3 ). The objective was to study the effect of a different roughness configuration than the distributed roughness.

In the anechoic tests four inflow velocities were used in each configuration, in order to have enough data to determine the velocity scaling of the noise spectra. The Reynolds numbers were $1.6 \times 10^{5}(20 \mathrm{~m} / \mathrm{s})$, $2.2 \times 10^{5}(27 \mathrm{~m} / \mathrm{s}), 2.7 \times 10^{5}(34 \mathrm{~m} / \mathrm{s})$, and $3.2 \times 10^{5}(40 \mathrm{~m} / \mathrm{s})$. In the low speed wind tunnel measurements the free stream velocity was limited to $27 \mathrm{~m} / \mathrm{s}\left(R e=2.2 \times 10^{5}\right)$ in the case of the on-surface microphone measurements and the phased microphone array measurements, and to $24 \mathrm{~m} / \mathrm{s}\left(R e=1.9 \times 10^{5}\right)$ in the case of the PIV measurements.

\section{Results and Discussion}

\section{A. Flat plate}

Measurements of the TBL thickness at various streamwise locations were performed using a Pitot tube, and the results are shown in figure 5, together with the prediction of the model by Krogstad et al. ${ }^{23}$ using the friction coefficient evolution model of Mills and Hang, ${ }^{25}$ and the law of the wall with a wake function, in the smooth configuration. It is observed that the thickness is underpredicted in the upstream region of the rough surface, where the transition between the smooth surface to rough surface happens. The predictions are improved at downstream locations for both the hemispherical and the cylindrical roughnesses.

The far field sound spectra measured at two observation angles for the highest flow speed are shown in figure 6. For frequencies lower than approximately $800 \mathrm{~Hz}$ all spectra collapse with the smooth wall data. 


\begin{tabular}{|l|ccc|}
\hline CONFIGURATION & $h(\mathrm{~mm})$ & $\sigma$ & $\zeta\left(^{\circ}\right)$ \\
\hline Smooth & 0 & - & - \\
SmoothNotTripped & 0 & - & - \\
\hline \hline Distributed roughness & & & \\
\hline Spheres4mm & 4 & 0.42 & - \\
Spheres4.5mm & 4.5 & 0.53 & - \\
Cylinders4mm & 4 & 0.42 & - \\
Cylinders4.5mm & 4.5 & 0.53 & - \\
\hline \hline Cable & & & \\
\hline Cable $4 \mathrm{~mm} 6$ & 4 & 0.05 & 79 \\
Cable $4 \mathrm{~mm} 15$ & 4 & 0.12 & 85 \\
Cable $4 \mathrm{~mm} 40$ & 4 & 0.33 & 88 \\
Cable6mm6 & 6 & 0.08 & 79 \\
Cable6mm15 & 6 & 0.19 & 85 \\
Cable6mm40 & 6 & 0.50 & 88 \\
\hline
\end{tabular}

Table 2. Roughness configurations.

A broadband peak is observed at all observation angles for the hemispherical elements, which scales well with $U_{o}^{6}$, i.e. has dipole-like character, as is described by the roughness noise prediction models. The peaks collapse on a Strouhal number basis at $f h / U_{o} \approx 0.18$. Howe's prediction model predicts the peak to be at $f h / u_{\tau} \approx 3$, which is equivalent to the above relation since in typical rough wall TBL's the value of $u_{\tau} / U_{o}$ lays approximately between 0.05 and $0.07 .{ }^{24}$ The peak corresponds to roughness noise and is very well predicted by Howe's model (figure 7). The two empirical constants of Smol'yakov model have been fixed to best fit the measurements at $\theta=121^{\circ}$, giving a good match with the experimental trends for this case $\left(K_{R}=6.36 \cdot 10^{-19}\right.$, and $S t_{w}=2$, i.e. $\left.f h / U_{o} \approx 0.16\right)$. However, at different observation angles it does not predict the levels well. The value of $S t_{w}=3.81$ determined by Smol'yakov ${ }^{11}$ causes the peak frequency to be too large (approximately 1.8 times larger than the measurements). This implies that the lack of directivity information of the model is a serious drawback for its use in practical applications. In the case of the cylindrical elements the patterns are significantly different. In the first place the roughness noise peak levels are lower than those of hemispherical roughness case. This is true especially for the two downstream observation angles. The spectral levels deviate from the hemispherical roughness case before the maximum peak is reached and start reducing with a fall-off rate close to that of the smooth wall spectra. The fact that at $\theta=121^{\circ}$ the peak is close to the hemispherical roughness case up to higher frequencies suggests that the noise generation mechanism is only partially modified.

At frequencies higher than the previously described roughness noise peak, the cylindrical roughness configurations have a strong broadband peak. The peak is not Strouhal-based; it is only weakly shifted to higher frequencies with the increasing outer velocity, and it has been determined to scale with $U_{o}^{7-8}$. Yang and Wang ${ }^{26-28}$ performed LES simulations of a TBL flow over a roughness patch of hemispherical, cylindrical and cuboidal elements with $h / \delta \approx 0.1$ and $\sigma=0.1$, and their results showed a monotonous increase of noise from $f h / U_{o} \approx 0.15$ for both the cylinders and cuboids, with respect to the hemispheres case. They observed that the turbulent surface pressure field was dominated by the flow structures in the vicinity of the front edges due to sharp edge separation. Also the pressure fluctuations were strongest in the leading row of roughness elements, and diminished downstream. The peaks are not detected in the reported simulation effort. Possible explanations for the observed peak in the present experiments are: a) it is due to interaction effects between neighbouring roughness elements, since the present roughness is more densely packed than the ones in the simulations ( $\sigma=0.42$ and $\sigma=0.1$, respectively.), b) the difference is related to the difference in roughness height to TBL thickness ratio $(h / \delta>0.4$ in the present experiments and $h / \delta=0.12$ in the simulations), and c) the simulations (LES) did not resolve the flow structures responsible for the measured noise (the peak frequency is around $11 \mathrm{kHz}$, which is close to the reported maximum frequency). Despite the differences found between the present experiments and the simulations, it is reasonable to suspect that 
the peak measured in the present experiments with the sharp edge separation phenomenon was the same as that observed in the simulations.

The measured noise directivity patterns are shown in figure 8. The values correspond to overall sound pressure levels obtained by integration of $P(\omega)$ between $1 \mathrm{kHz}$ and $3 \mathrm{kHz}$ (figure $8(\mathrm{a})$ ) and between $3 \mathrm{kHz}$ and $18 \mathrm{kHz}$ (figure $8(\mathrm{~b})$ ). The roughness noise peak has a minimum at $\theta=90^{\circ}$, as is predicted by the model, and in that observation angle the background noise is larger than the predicted levels. The directivity of the high frequency noise peak of the cylindrical roughness has a directivity pattern with a maximum at $90^{\circ}$, and close to a transverse dipole $\left(P(\omega) \propto \sin ^{2} \theta\right)$.

The $\triangle \mathrm{SPL}$ plots obtained by subtracting the smooth wall noise levels from the rough wall levels are shown in figure 9. It can be appreciated that the roughness noise is more pronounced in the upstream and downstream directions, as was described by Liu and Dowling. The sharp-edge noise peak is most pronounced in the upstream direction and its level diminishes in the downstream direction.

A configuration corresponding to a larger value of $h / \delta$ in the leading row of elements was tested to determine the effect of this parameter on the radiated noise. In the baseline configurations the roughness height to TBL thickness ratio in the leading row of elements is, based on the smooth wall TBL thickness, $h / \delta \approx 0.5$. In the additional configuration the distance from the leading edge of the plate to the first row of roughness elements was reduced by half, resulting in $h / \delta \approx 1$. The far field measurements of both configurations are shown in figure $6(\mathrm{~d})$ for one observation angle. The difference between the two cases is small, which implies that the far field noise dependence on $h / \delta$ is weak, and therefore the flow surrounding the leading rows of elements is not a dominant source of noise. This is true for both hemispherical and cylindrical elements in all the observed frequency range.

\section{B. Circular cylinder}

\section{Roughness noise}

The far field spectra shown in figures 10 and 11 reveal a broadband peak in the distributed roughness configurations that, is especially prominent for the Spheres $4 \mathrm{~mm}$ and Spheres $4.5 \mathrm{~mm}$ cases, similar to the flat plate cases. It appears at higher frequencies than for the flat plate cases, but it is Strouhal-based with the peak around $S t \approx 10$. The spectra scale well with $U_{o}^{6}$. However in this case there isn't a unique value of outer velocity; the outer velocity changes along the TBL. In the flat plate configuration the roughness noise peak is located at $f h / U_{o} \approx 0.2$. If the frequency is normalized with the maximum outer velocity around the cylinder instead of the free stream velocity $\left(U_{\max } \approx 1.7 U_{o}\right.$ based on PIV measurements), and the roughness height instead of the cylinder diameter we can write:

$$
\frac{f h}{U_{\max }}=\frac{f D}{U_{o}} \cdot \frac{U_{o}}{U_{\max }} \cdot \frac{h}{D} \approx 10 \cdot \frac{40}{1.7 \cdot 40} \cdot \frac{4}{127} \approx 0.18
$$

i.e. the same value obtained for a flat plate when normalizing with the free stream velocity instead of the maximum outer velocity (they are the same in the flat plate case). This suggests that most of the noise comes from the region of maximum outer velocity.

In figures 10 and 11 the spectra of the cylindrical roughness are also shown. They have a significantly less pronounced peak than the hemispheres. The noise generation mechanism is weakened for this case, and the weakening is stronger for the larger cylinders. Two possible explanations for this discrepancy between the hemispherical and the cylindrical roughness can be proposed. Assuming the dominant turbulent stresses are similar in both configurations, the scattering phenomenon is more efficient at generating noise in the case of the hemispheres. In this case the use of a Green's function tailored to cylindrical roughness instead of hemispherical roughness would predict the noise correctly. On the other hand, the difference may be due to differences in strength or location of the turbulent stresses. Despite the fact that the heights of the roughness elements are the same for both configurations, the cylindrical roughness elements have a higher blockage and a higher solidity (projected frontal roughness area per unit wall parallel area) than the hemispheres and the dominant Reynolds stresses may be shifted significantly away from the roughness elements, diminishing the noise generation efficiency. Further studies are required to clarify the precise mechanism(s).

For frequencies larger than the peak described above, the cylindrical roughness has higher spectral levels than the hemispheres, with the exception of Spheres $4 \mathrm{~mm}$ at $\theta=46^{\circ}$, which has similar levels as the cylindrical configurations. As in the flat plate case, this difference may be due to sharp edge separation, however the increase in this case is much smaller than what is observed for the flat plate case. The sharp edge noise in 
the flat plate case mainly happens when the incoming thin smooth-wall TBL impinges on the leading rows of roughness elements, as was shown by Yang and Wang. ${ }^{27,28}$ In the circular cylinder this doesn't occur because in the region of higher outer velocity the TBL has already adapted to the rough surface, and it is already thick. Furthermore, in the flat plate case the peak scales with $U_{o}^{7-8}$ and in the circular cylinder with $U_{o}^{6-7}$. This implies that the flow structures responsible for the noise generation are different for both cases.

A set of phased microphone array measurements were performed for the distributed roughness configurations on the circular cylinder (see figures 12 and 13), to obtain information regarding the location of the noise sources. The source strength maps show that within the frequency range in which roughness noise is dominant the most powerful sources are located on the upstream face of the cylinder and the sides. Figure 12 shows the source strength maps at $f=1.9 \mathrm{kHz}$. The rough cylinders emit more noise at this frequency, and the sources are located on the cylinder surface, mainly around the separation lines. From the far field spectra it is seen that at this frequency roughness noise starts to dominate. The Spheres $4 m m$ and Cylinders $4 \mathrm{~mm}$ cases have very similar source maps, as was expected from the far field spectra at this frequency. In figure 13 the maps at $f=3 \mathrm{kHz}$ are presented. As in the previous case, noise levels of the rough cylinders are above the smooth case. At this frequency the strongest sources are located mainly on the upstream face of the cylinder, and not in the separation region. At this frequency the hemispherical roughness configuration radiates more intensely than the cylindrical roughness configuration, as observed in the far field spectra as well. It can also be observed that the highest contribution comes from the spanwise extents of the cylinder, due to the interaction of the TBL's growing on the endplates with the rough surface of the cylinder extents.

Figure 14 shows the spectra of the on-surface microphones for the distributed roughness configurations. The baseline case to which they have been compared is SmoothNotTripped. No available data of Smooth exist at the same locations as the rough cases. At the downstream stagnation line the spectral levels of the rough cylinders are of the order of $10 \mathrm{~dB}$ higher than the smooth case, for $7<S t<30$. This difference appears constant in the spectra, and no sign of a peak similar to the far field case is observed. Hemispherical roughness and cylindrical roughness have very similar levels in all the frequency range. At $\theta=40^{\circ}$ the spectrum of the smooth cylinder approximately collapses with the rough cases in $6<S t<10$, but for $S t>10$ its levels are lower. The microphone at $\theta=120^{\circ}$ is located within the upstream attached flow. Discernible differences appear between the various roughness configurations. The peak Strouhal numbers of the hemispherical roughness are larger than the cylindrical roughness and their spectral levels are significantly higher in the range $8<S t<60$. In both hemispherical and cylindrical roughness the peak Strouhal numbers are bigger for the larger roughness elements. Finally the peak levels are approximately $3 \mathrm{~dB}$ higher for the smaller roughness sizes. This difference is explained by the effect of the various roughness geometries on the TBL structure. Higher roughness size implies a larger distance from the dominant stresses to the microphone, and therefore the levels are lower. The main differences between hemispherical and cylindrical roughness are, as was mentioned earlier, the blockage and the sharp edges. The higher blockage of the cylindrical roughness moves the Reynolds stresses profiles away from the wall, which may explain the significantly lower levels for $S t>8$.

The far field spectra of the cable configurations also present a broadband peak, as can be observed in figures 10 (b) and 11 (c,d), but at lower frequencies than the cylinders with distributed roughness. It is located around a Strouhal number of 0.7, although due to its weakness compared to the background levels it is not clear whether it is Strouhal-based or not. The peak seems to scale approximately with $U_{o}^{6}$ for all configurations. The far field noise measurements of Hutcheson and Brooks ${ }^{16}$ on cylinders with large cable wrapped helicoidally showed a broadband peak at a frequency that corresponds to a Strouhal number of 0.7 , as in this case, but the velocity and frequency scalings weren't reported. Their cable diameter was such that $h / D$ is 0.25 , i.e. much larger than the configurations considered here. Hutcheson and Brooks observed that, when the peak frequency was scaled with the cable diameter and the incoming velocity, a Strouhal number close to 0.2 was obtained, typical of a vortex shedding mechanism. However it is known that flow around a circular cylinder sufficiently close to a wall could suppress vortex shedding. ${ }^{29}$ If the peak is associated with cable-induced roughness noise and not with a larger scale phenomena induced by the cable, this noise must be originated from the scattering of the turbulent stresses perpendicular to the cable. Further experiments are required to clarify the origin of this peak.

It can be observed that, at all Reynolds numbers and cable configurations, the spectra collapse for frequencies larger than $S t \approx 2$ up to frequencies of $S t \approx 50$, to within $\pm 2 \mathrm{~dB}$ (figure 11). The collapse implies that the presence of the cable doesn't affect the noise generation in this frequency range. In the two configurations with lower density of cable it is reasonable to assume that the cable, being parallel to 


\begin{tabular}{|l|cccc|}
\hline CONFIGURATION & $f \approx f_{v s}$ & $f_{v s} \ll f \ll c_{o} / D$ & $f \approx f_{r n}>\approx c_{o} / D$ & $f \gg c_{o} / D$ \\
\hline Endplates & 5 & 5.5 & - & $5.5-6$ \\
Smooth & 6 & $6.5-7$ & - & 6.5 \\
SmoothNotTripped & - & 7 & - & $6.5-7$ \\
Spheres4mm & 6 & $6.5-7$ & 6 & $7-7.5$ \\
Spheres4.5mm & 6 & $6.5-7$ & 6 & $6.5-7$ \\
Cylinders4mm & 6 & 6.5 & 6 & $6-6.5$ \\
Cylinders4.5mm & 6 & $6-6.5$ & 6 & 6 \\
Cable_mm6 & - & 7 & - & $6-6.5$ \\
Cable4mm15 & - & $6.5-7$ & - & $5.5-6$ \\
Cable4mm40 & - & $6.5-7$ & - & $6-6.5$ \\
Cable6mm6 & - & $7-7.5$ & - & $6.5-7$ \\
Cable6mm15 & - & $6.5-7$ & - & $6.5-7$ \\
Cable6mm40 & 6 & $7-7.5$ & - & 6.5 \\
\hline
\end{tabular}

Table 3. Inflow velocity scaling exponents for all configurations, determined from the signals measured at $\theta=135^{\circ} . f_{v s}$ and $f_{r n}$ are the vortex shedding and the roughness noise peak frequencies, respectively.

the mean flow, doesn't cause transition to occur, so that the flow around the cable on the upstream face is laminar and therefore lower roughness noise is generated (without transition strips the Reynolds numbers tested correspond to the critical regime, in which transition occurs at the separation region). However, in the configuration with dense cable density it is not reasonable to think that the flow is laminar over the upstream face. Therefore, the collapse implies that the scattering of turbulent sources on the cable is inefficient at generating noise.

\section{Simple roughness noise model for circular cylinders}

Despite the generation of noise due to TBL-roughness interaction being analogous in flat plates and circular cylinders, the propagation of sound to the far field is different in both situations. A simple extension of Howe's model has been used to predict the roughness noise of the circular cylinder configurations, using the following simplifications:

a. The roughness noise is generated only on the upstream face of the cylinder. The detachment line is approximately located at $\theta=90^{\circ}$, and the flow velocity at the downstream face is typically much smaller.

b. The TBL model accounts for the increase of outer velocity by introducing a distribution $U_{o}(x)$ instead of the constant freestream velocity in the equations that determine the evolution of $c_{f}(x), u_{\tau}(x)$ and $\delta(x)$ on a flat wall. This is equivalent to assuming that the TBL structure adapts instantaneously to the changing outer velocity and without a pressure gradient.

c. Noise is scattered from each area element as if the surface was a flat wall, parallel to the cylinder in that element (see figure 17).

In figure 18 the comparison between measurements and predictions is shown for three observation angles. The noise peak frequency is well predicted at both speeds. The peak levels are over-predicted by 2 to 3 $\mathrm{dB}$ at the three highest observation angles, and the difference increases at higher frequencies. At $\theta=28^{\circ}$ the peak level is overpredicted by 8 to $10 \mathrm{~dB}$. The reasons for the overprediction are possibly due to the three assumptions used to derive the model. In the first place it is known that smooth and rough TBL's under a FPG are thinner and have weaker Reynolds stresses. ${ }^{30}$ Therefore the assumption of ZPG TBL can cause overprediction of the noise levels. Secondly, the assumption of TBL detachment at $90^{\circ}$ may as well be a source of error. From the PIV measurements it's not possible to determine the exact separation point. However, as was observed previously, the effect of roughness is to cause an early detachment. According 
to the measured Strouhal number of the vortex shedding peak, which is close to the subcritical value, and the low spanwise coherence in the supercritical and postcritical regimes, it is likely that the detachment point is located at $\phi<90^{\circ}$. This could also be a cause of overprediction. Finally, the possible influence that the assumption of a flat wall may have in the propagation of sound to the far field is complicated. In figure 17 the flat wall directivity pattern on each rough wall area element is shown. It is a streamwise dipole aligned with the wall. This pattern may be significantly different in the case of cylindrical geometry. Further development of the model is required to improve its predictive accuracy.

\section{Vortex shedding}

The far field spectra measured at $\theta=46^{\circ}$ is shown in figure 10. The tripped smooth configuration presents a vortex shedding peak with a Strouhal number $S t \approx 0.26$, which is in agreement with previously reported highReynolds number measurements ${ }^{13}$ and indicates that the tripping is successful. The distributed roughness cases also have vortex shedding peaks, but at significantly smaller Strouhal numbers than the smooth case. In the case of distributed roughness $S t$ is approximately 0.20 , with little difference between hemispherical and cylindrical roughness. It was seen in past experiments that cylinders with roughness as large as $h / D=$ 0.03 had strong coherent vortex shedding with values of Strouhal number and drag coefficient close to the subcritical regime values. The mean drag appeared to keep increasing with $h / D$ up to the highest tested roughness size. The present roughness configurations follow this trend. The explanation was due to the effect that roughness had within the TBL's. According to Guven et al. ${ }^{15}$ roughness causes the TBL's to be thicker and with a higher momentum deficit, which implies an earlier separation than the smooth cylinder and a wider wake. This explains the decrease of Strouhal number. The mean velocity fields obtained using PIV (figure 15) reveal a wider wake immediately after separation for the rough cylinders, in agreement with the previous argument. The RMS velocity fields of the streamwise stresses (figure 16) show remarkably higher levels of turbulent fluctuations in the shear layers and the near wake of the rough cylinders. According to Zdravkovich ${ }^{17}$ for coherent vortex shedding to be present it is required that there is homogeneous separation along the span. That appears to be the case in the present distributed roughness sizes.

The on-surface microphone measurements as shown in figure 14 indicate clearly the presence of the vortex shedding peaks in the distributed roughness configurations. The peak Strouhal number is 0.2 , the same value measured in the far field acoustic signals. At the downstream stagnation point the first harmonic, corresponding to the alternating drag force, is more intense than the fundamental one. In the other locations the fundamental peak is dominant over the first and second harmonics. This indicates that the pressure fluctuations related with the oscillating lift, at the vortex shedding frequency are much stronger close to the separation region than close to the stagnation line, and the opposite happens for the pressure fluctuations associated with the oscillating drag, at twice the vortex shedding frequency.

The cable configuration with the highest surface density (Cable6mm40) has a weak but discernible vortex shedding peak at $S t \approx 0.22$, a value between the smooth cylinder and the cylinders with distributed roughness. It is known that dense circumferential grooves have the same effect on the mean drag and vortex shedding frequency as distributed roughness. ${ }^{18}$ In the present case the results suggest that the transition to the flow regime corresponding to distributed roughness has only partially occurred.

All vortex shedding peaks scale approximately with $U_{o}^{6}$. For frequencies between $f_{v s}$ and $c_{o} / D$, corresponding to the fall-off range between the vortex shedding peaks and the roughness noise dominated region, the various configurations show different scaling laws, all of them between $U_{o}^{6}$ and $U_{o}^{7.5}$.

\section{Conclusion}

The effect of large roughness with high surface density on the noise emitted by circular cylinders has been shown to be important over all the frequency range of the far field noise spectra. At high frequencies the hemispherical roughness generates noise well described by Howe's model. However, to correctly determine the far field noise a modification to account for the cylindrical geometry is needed. Here a simple extension based on outer potential flow and ZPG TBL has been used, which overpredicts the peak spectral levels, especially in the downstream direction, but predicts the peak frequency well. Cylindrical roughness has weaker roughness noise peak at $S t \approx 10$ compared to the hemispherical roughness, but it radiates higher noise levels at higher frequencies. This increase has been hypothesized to be due to sharp-edge separation.

The effect of roughness on lower frequencies is to increase the noise levels due to the presence of stronger 
vortex shedding peaks, together with a fall-off rate similar to the smooth cylinder. The velocity scaling of the vortex shedding and spectral fall-off regions is similar to the smooth cylinder case, i.e. the vortex shedding peak scales with $U^{6}$ and the fall-off scales with $U^{6-7}$. The vortex shedding peak Strouhal number is lower than the smooth cylinder case, and close to the subcritical regime value. This fact, together with the increase of the peak levels is in agreement with other earlier experimental studies with similar roughness sizes, but different roughness shapes. They are successfully explained by the TBL thickening effect of roughness, which causes early separation and therefore a wider wake and a lower Strouhal number.

\section{Acknowledgments}

The research is supported by Airbus. The authors would like to thank Dr. Nicolas Molin for his advice and assistance during the course of the study. We also would like to thank especially Phil Oxborrow, Steve White, Chris Oliver, David Marshall, Mike Thomas and David Cardwell, for their help during the experiments.

\section{References}

${ }^{1}$ Guo, Y., "A Component-Based Model for Aircraft Landing Gear Noise Prediction", Journal of Sound and Vibration, Vol. 312, 2008, pp. 801-820.

${ }^{2}$ Fink, M. R., "Noise Component Method for Airframe Noise", Journal of Aircraft, Vol. 16, No. 10, 1979, pp. 659-665.

${ }^{3}$ Smith, M. G. and Chow, L. C., "Validation of a Prediction Model for Aerodynamic Noise from Aircraft Landing Gear", AIAA 2002-2581, $8^{\text {th }}$ AIAA/CEAS Aeroacoustics Conference, 17-19 June, Breckenridge, Colorado, 2002.

${ }^{4}$ Howe, M. S., Theory of Vortex Sound, Cambridge University Press, 2003.

${ }^{5}$ Howe, M. S., "On the Generation of Sound by Turbulent Boundary Layer Flow Over a Rough Wall", Proceedings of the Royal Society of London, Series A, Vol. 395, 1984, pp. 247-263.

${ }^{6}$ Liu, Y. and Dowling, A. P., "Assessment of the Contribution of Surface Roughness to Airframe Noise", AIAA Journal, Vol. 45, No. 4, 2007, pp. 855-869.

${ }^{7}$ Liu, Y., Dowling, A. P. and Shin, H.-C., "Measurement and Simulation of Surface Roughness Noise Using Phased Microphone Arrays", Journal of Sound and Vibration, Vol. 314, 2008, pp. 95-112.

${ }^{8}$ Ahn, B., "Modeling Unsteady Wall Pressures Beneath Turbulent Boundary Layers". PhD Thesis, Department of Engineering, University of Cambridge, 2005.

${ }^{9}$ Corcos, G. M., "The Structure of the Turbulent Pressure Field in Boundary-Layer Flows", Journal of Fluid Mechanics, Vol. 18, No. 3, 1964, pp. 353-378.

${ }^{10}$ Jimenez, J., "Turbulent Flows Over Rough Walls", Annual Review of Fluid Mechanics, Vol. 36, 2004, pp. 173-196.

${ }^{11}$ Smol'yakov, A. V., "Noise of a Turbulent Boundary Layer Flow over Smooth and Rough Plates at Low Mach Numbers", Acoustical Physics, Vol. 47, No. 2, 2001 pp. 218-225.

${ }^{12}$ Schlichting, H. and Gersten, K., Boundary Layer Theory, Springer, 8th Edition, 2000.

${ }^{13}$ Achenbach, E. and Heinecke, E., "On Vortex Shedding from Smooth and Rough Cylinders in the Range of Reynolds Numbers 6000 to 5000000", Journal of Fluid Mechanics, Vol. 109, 1981, pp. 239-251.

${ }^{14}$ Achenbach, E., "Influence of Surface Roughness on the Cross-Flow Around a Circular Cylinder", Journal of Fluid Mechanics, Vol. 46, 1971, pp. 321-335.

${ }^{15}$ Guven, O., Farell, C. and Patel, V. C., "Surface Roughness Effects on the Mean Flow Past Circular Cylinders", Journal of Fluid Mechanics, Vol. 98, 1980, pp. 673-701.

${ }^{16}$ Hutcheson, F. V. and Brooks, T. F., "Noise Radiation from Single and Multiple Rod Configurations", AIAA 2006-2629, $12^{\text {th }}$ AIAA/CEAS Aeroacoustics Conference, 2006.

${ }^{17}$ Zdravkovich, M. M., Flow Around Circular Cylinders. Vol. 1: Fundamentals, Oxford Science Publications, 1997.

${ }^{18}$ Zdravkovich, M. M., Flow Around Circular Cylinders. Vol. 2: Applications, Oxford Science Publications, 1997.

${ }^{19}$ Zdravkovich, M. M., "Review and Classification of Various Aerodynamic and Hydrodynamic Means for Suppressing Vortex Shedding", Journal of Wind Engineering and Industrial Aerodynamics, 1981, pp. 145-189.

${ }^{20}$ Chong, T. P., Joseph, P. F. and Davies, P. O. A. L., "Design and Characterisation of a Quiet, Low Turbulence Open Jet Blow Down Wind Tunnel in ISVR", ISVR Technical Report, No. 322, 2008.

${ }^{21}$ Castro, I. P., "Hot Wire Anemometry with the Newcastle, NSW bridges", University of Southampton, Aerodynamics and Flight Mechanics Research Group, 2001.

${ }^{22}$ Fenech, B. A. and Takeda, K., "SotonArray: Southampton University Wind Tunnel Microphone Array System Guide", AFM Technical Report 07/03, School of Engineering Sciences, University of Southampton, 2003.

${ }^{23}$ Krogstad, P. A., Antonia, R. A. and Browne, L. W. B., "Comparison Between Rough- and Smooth-Wall Turbulent Boundary layers", Journal of Fluid Mechanics, Vol. 245, 1992, pp. 599-617.

${ }^{24}$ Howe, M. S., "The Turbulent Boundary-Layer Rough-Wall Pressure Spectrum at Acoustic and Subconvective Wavenumbers", Proceedings of the Royal Society of London, Series A, Vol. 415, 1988, pp. 141-161.

${ }^{25}$ Mills, A. F. and Hang, X., "On the Skin Friction Coefficient for a Fully Rough Flat Plate", Journal of Fluids Engineering, Vol. 105, No. 3, 1983, pp. 364-365. 
${ }^{26}$ Yang, Q. and Wang, M., "Computational Study of Roughness-Induced Boundary-Layer Noise", AIAA Journal, Vol. 47, No. 10, 2009, pp. 2417-2429.

${ }^{27}$ Yang, Q. and Wang, M., "Boundary-Layer Noise Induced by a Roughness Patch", AIAA 2010-3772, $16^{\text {th }}$ AIAA/CEAS Aeroacoustics Conference, 2010.

${ }^{28}$ Yang, Q. and Wang, M., "Statistical Analysis of Acoustic-Source Field in Rough-Wall Boundary Layers", AIAA 20112738, $17^{\text {th }}$ AIAA/CEAS Aeroacoustics Conference, 2011.

${ }^{29}$ Bearman, P. and Zdravkovich, M. M., "Flow Around a Circular Cylinder Near a Plane Boundary", Journal of Fluid Mechanics, Vol. 89, No. 1, 1978, pp. 33-47.

${ }^{30}$ Cal, R. B., Brzek, B., Johansson, T. G. and Castillo, L., "The Rough Favourable Pressure Gradient Turbulent Boundary Layer", Journal of Fluid Mechanics, Vol. 641, 2009, pp. 129-155. 


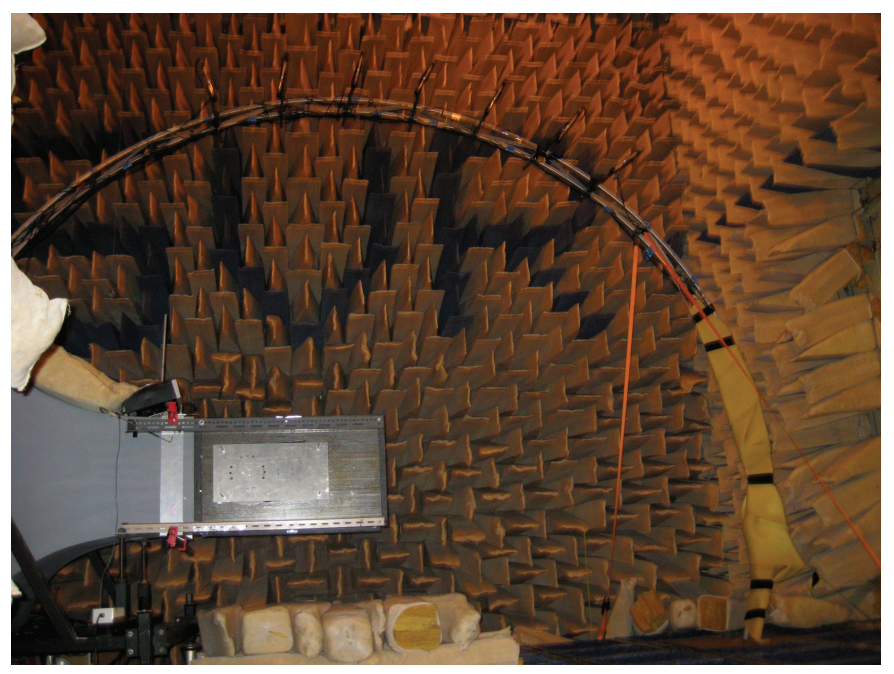

Figure 1. Circular cylinder and flat plate arrangement in the anechoic chamber.

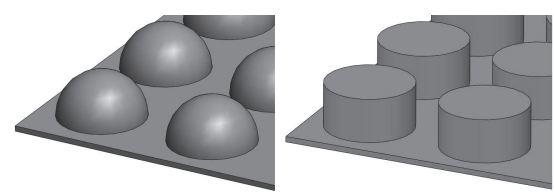

Figure 2. Distributed roughness configurations: Spheres $4 \mathrm{~mm}$ (left) and Cylinders $4 m m$ (right).

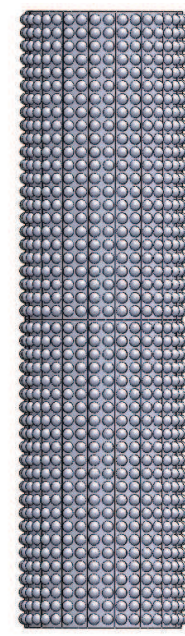

(a)

Spheres $4.5 \mathrm{~mm}$

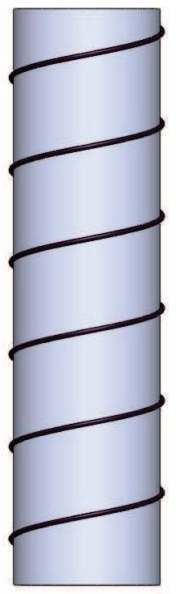

$\mathrm{n}=6$

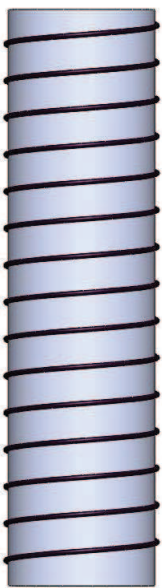

$\mathbf{n}=15$

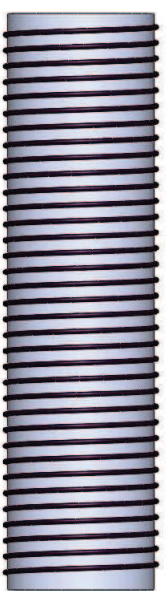

$\mathrm{n}=40$

(b) Cable6mm6, Cable6mm15, Cab6mm40

Figure 3. Rough circular cylinders. 


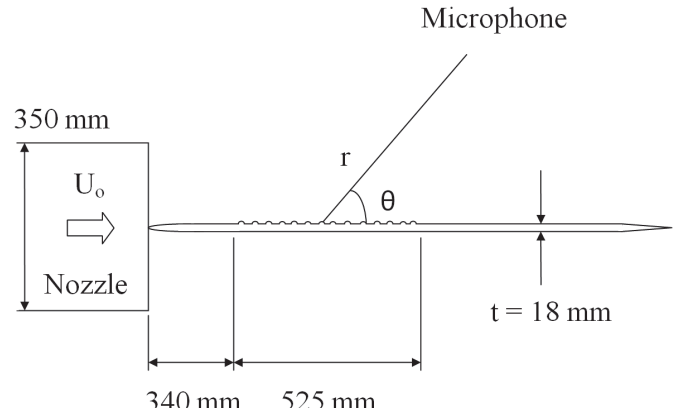

(a) Flat plate

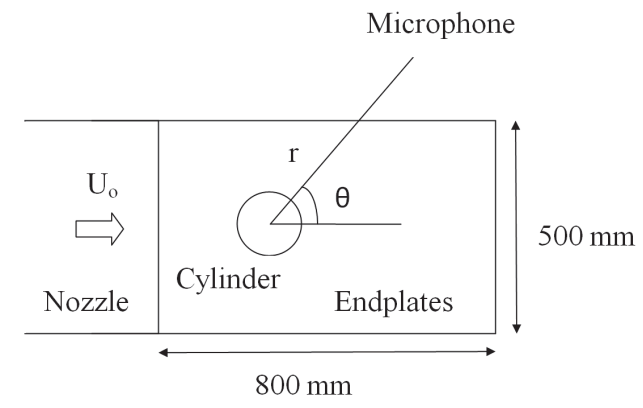

(b) Circular cylinder

Figure 4. Observation angle in the acoustic measurements.

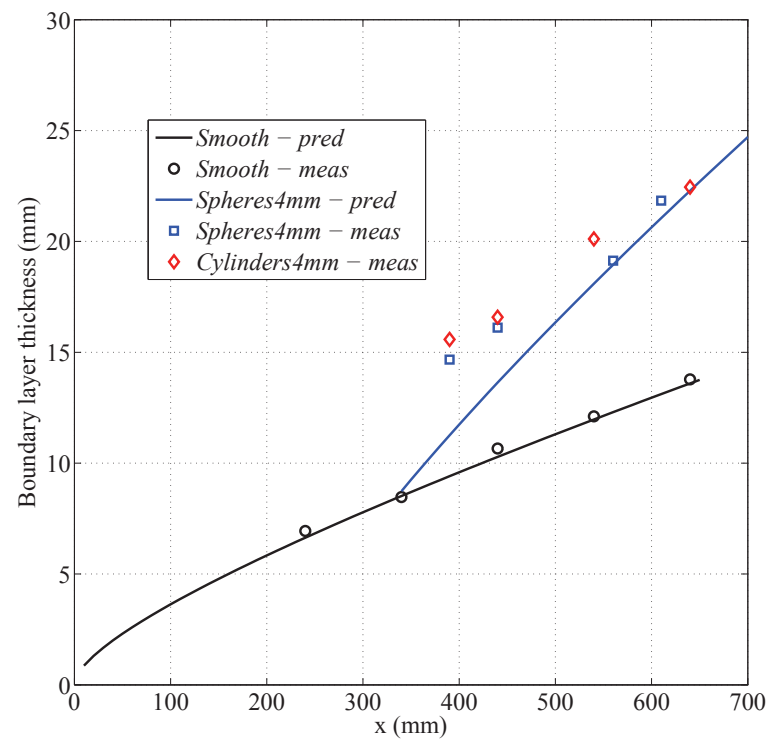

Figure 5. Boundary layer thickness evolution measured in the closed section wind tunnel. 


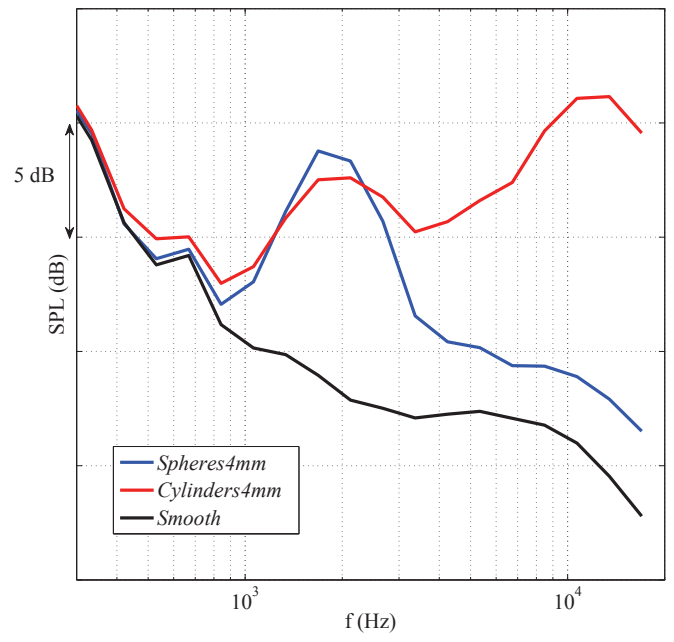

(a) Upstream elements fully covered by the boundary layer, $\theta=121^{\circ}$

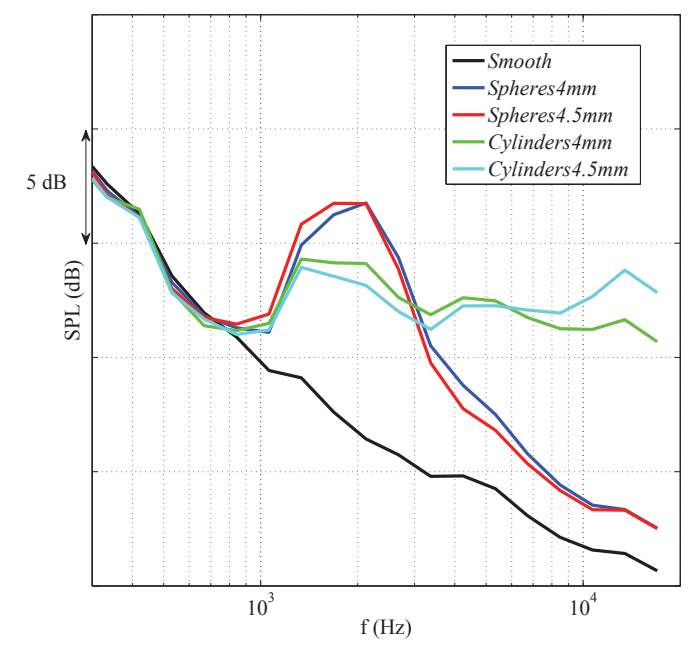

(b) Upstream elements fully covered by the boundary layer, $\theta=48^{\circ}$

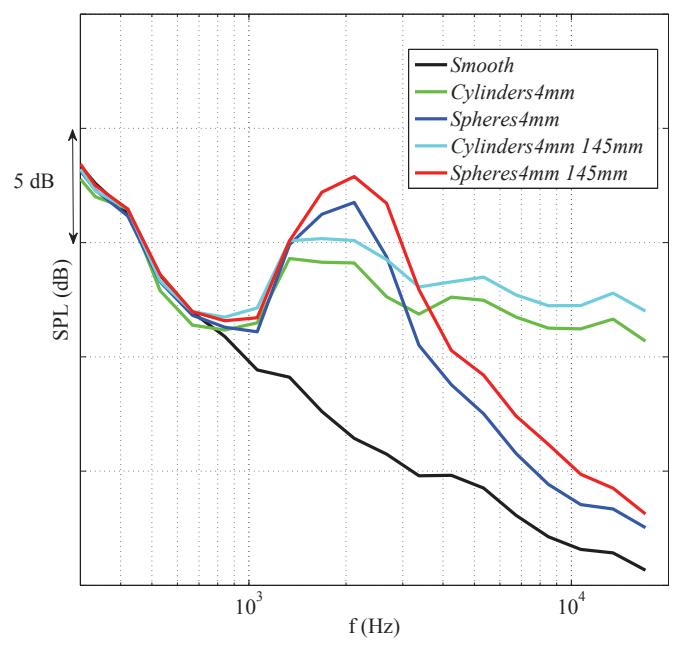

(c) Effect of $h / \delta$ at the upstream row of roughness elements, $\theta=48^{\circ}$

Figure 6. 1/3-octave band spectra of the far field noise of all rough flat plate configurations at $U_{o}=40 \mathrm{~m} / \mathrm{s}$. 


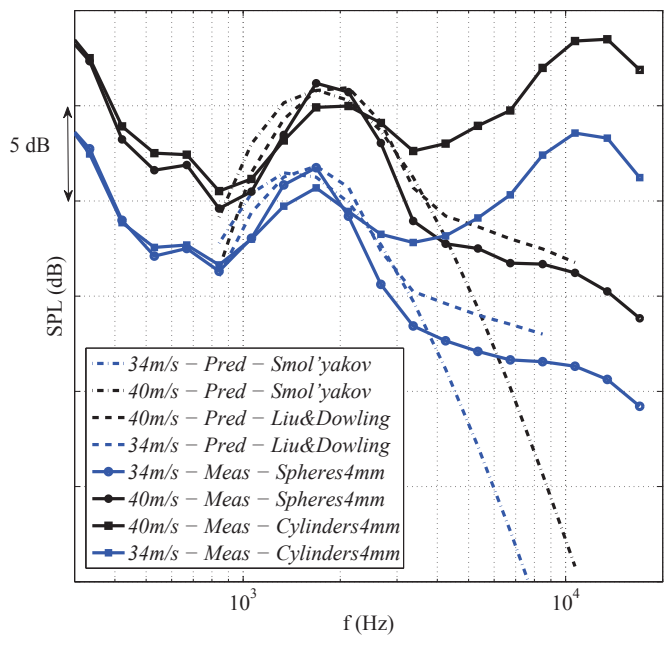

(a) $\theta=121^{\circ}$

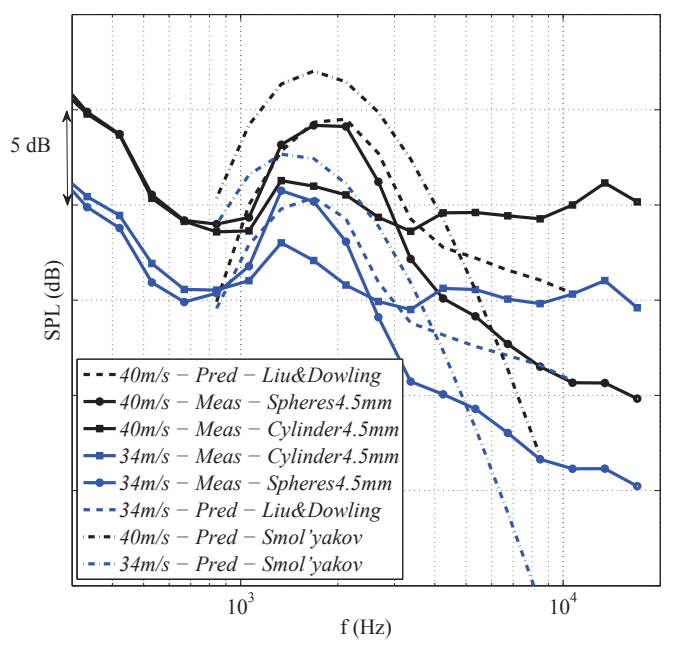

(b) $\theta=48^{\circ}$

Figure 7. Measurements and predictions of the far field noise 1/3-octave band spectra for the flat plate.

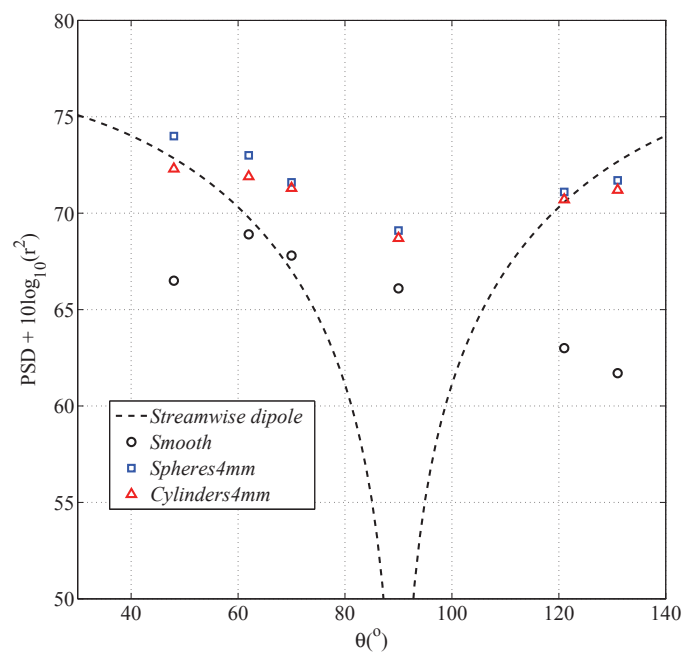

(a) OASPL between $1-3 \mathrm{kHz}$

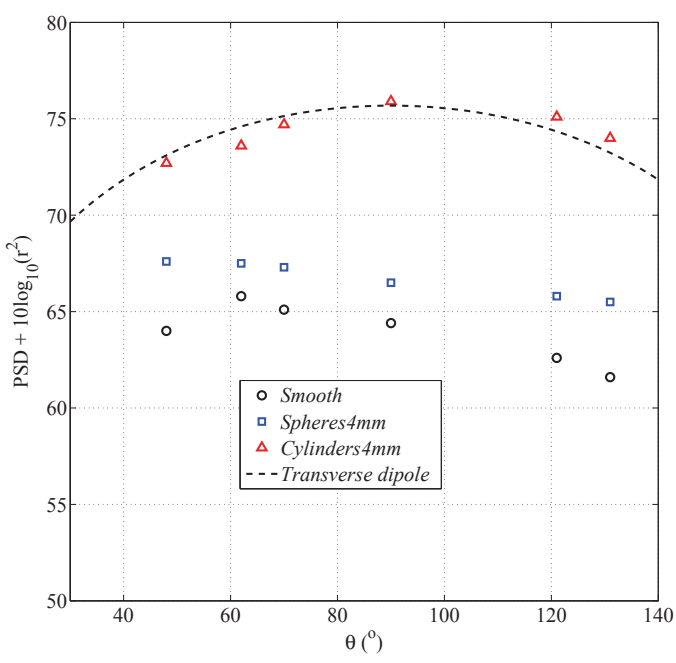

(b) $O A S P L$ between $3-18 \mathrm{kHz}$

Figure 8. Measured directivity patterns for the flat plate, obtained at $U_{o}=40 \mathrm{~m} / \mathrm{s}$. 


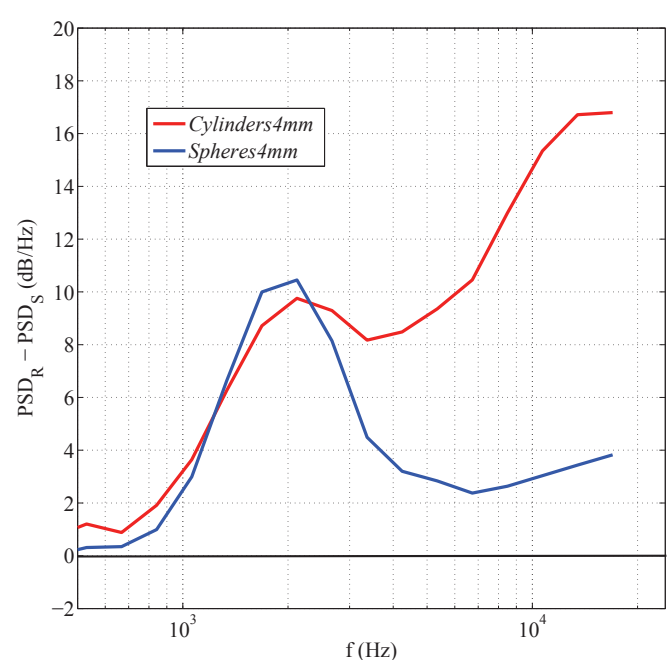

(a) $\theta=121^{\circ}$

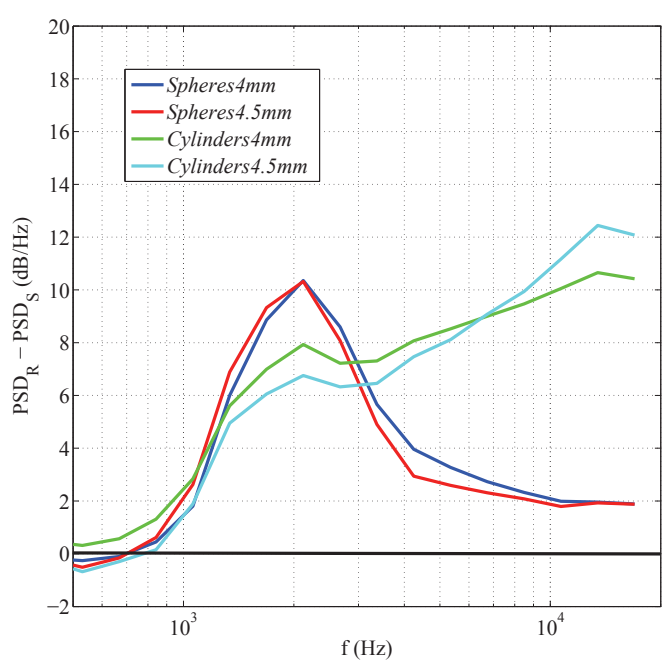

(b) $\theta=48^{\circ}$

Figure 9. $\triangle \mathrm{PSD}$ spectra of the flat plate roughness noise

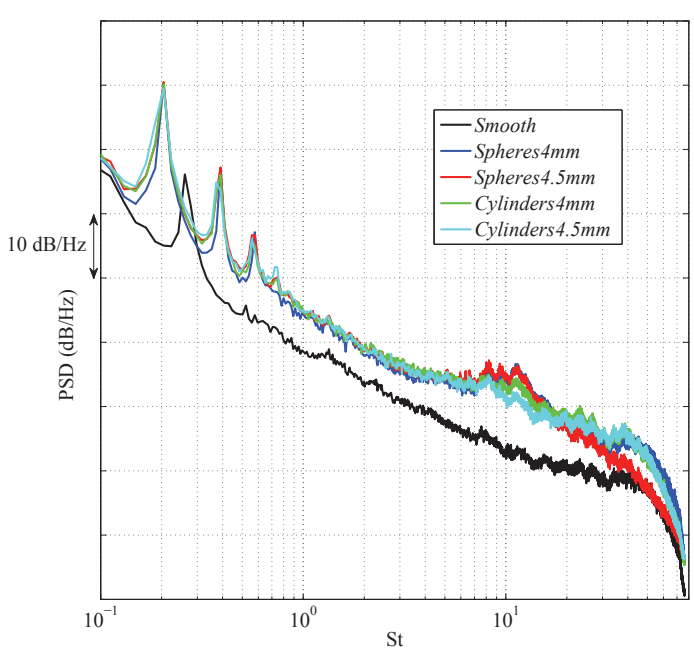

(a) Cylinders with distributed roughness

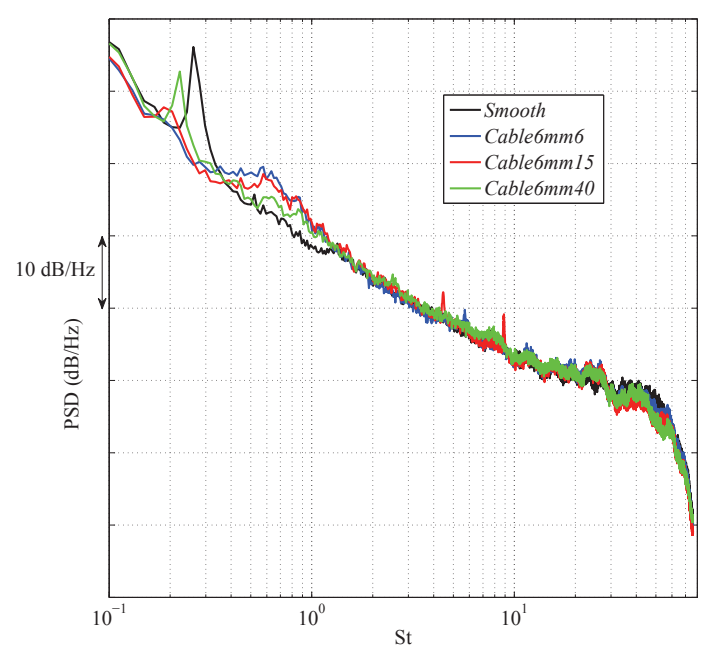

(b) Cylinders with $6 \mathrm{~mm}$ helicoidal cable

Figure 10. Far field noise of the rough circular cylinders measured for $R e=3.2 \cdot 10^{5}$ and $\theta=46^{\circ}$. 


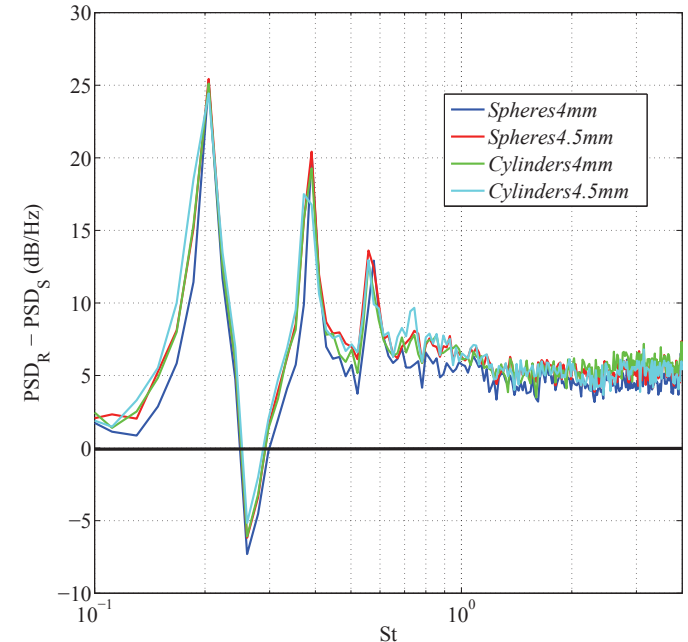

(a) Cylinders with distributed roughness in the low- and mid-frequency range

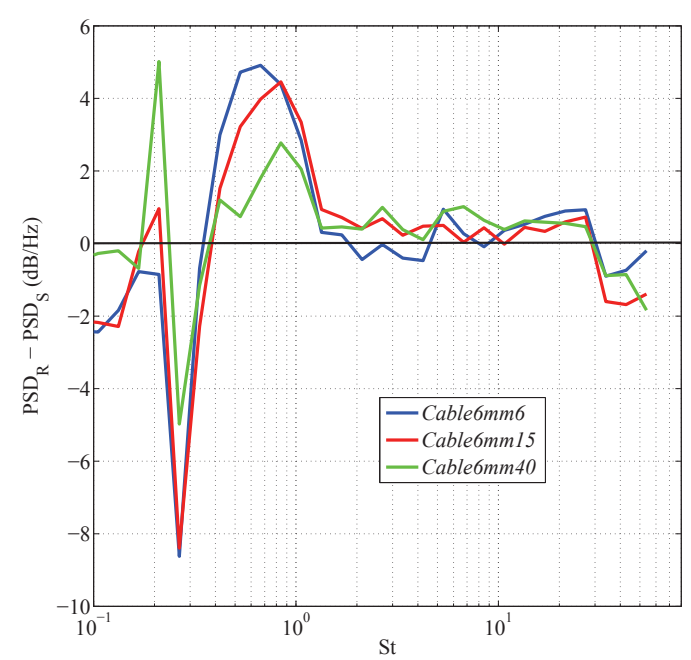

(c) Cylinders with $6 \mathrm{~mm}$ helicoidal cable

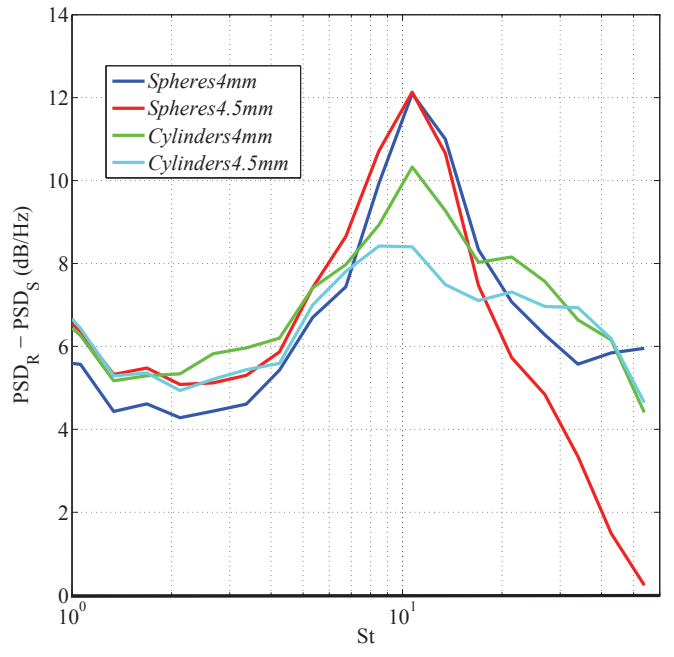

(b) Cylinders with distributed roughness in the highfrequency range

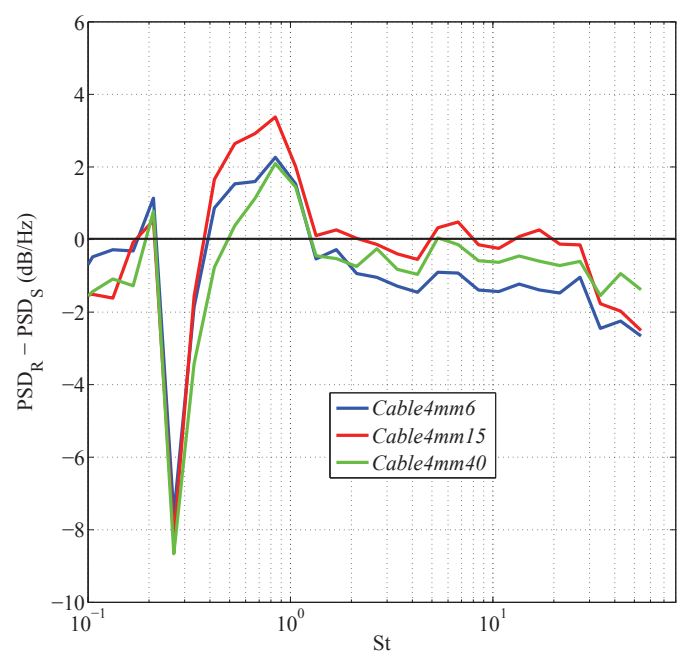

(d) Cylinders with $4 \mathrm{~mm}$ helicoidal cable

Figure 11. $\triangle$ PSD spectra of the rough cylinders for $R e=3.2 \cdot 10^{5}$ and $\theta=46^{\circ}$. 


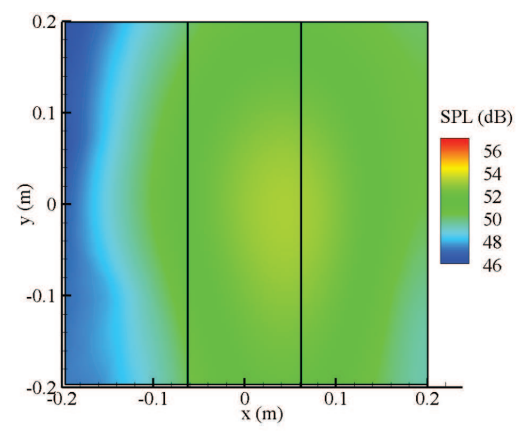

(a) Smooth

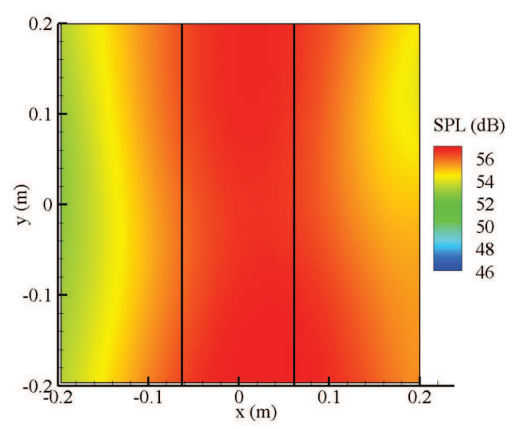

(b) Spheres4mm

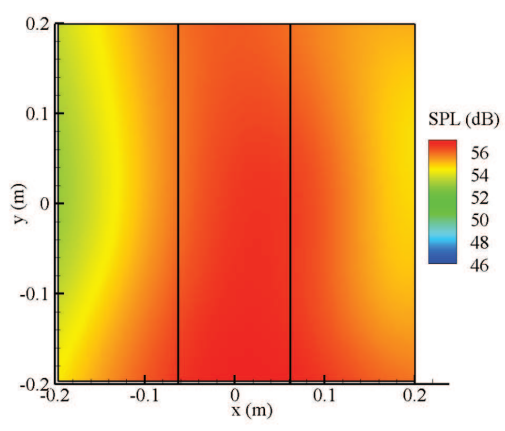

(c) Cylinders $4 \mathrm{~mm}$

Figure 12. Source strength maps at $f=1.9 \mathrm{kHz}$.

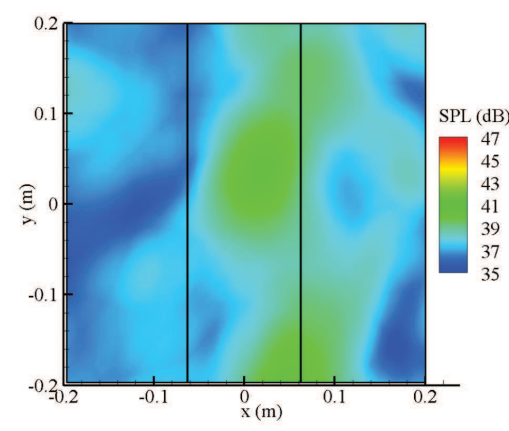

(a) Smooth

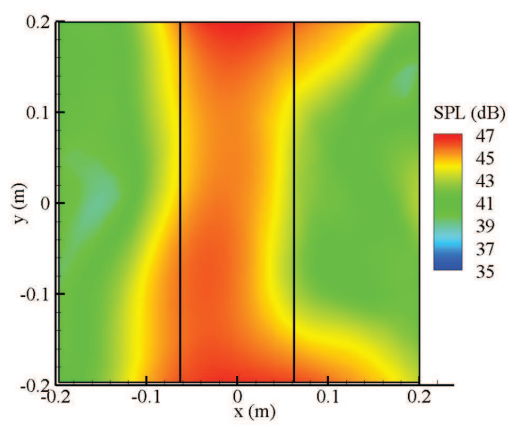

(b) Spheres4mm

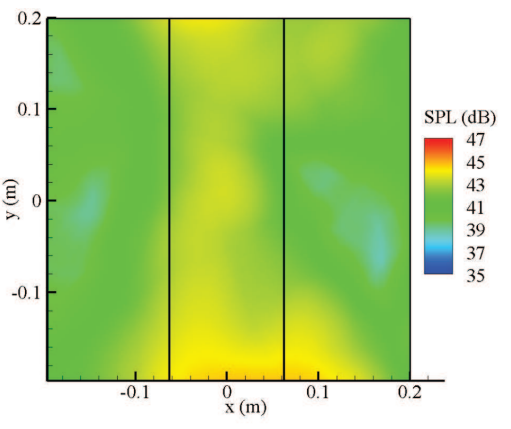

(c) Cylinders $4 \mathrm{~mm}$

Figure 13. Source strength maps at $f=3 \mathrm{kHz}$. 


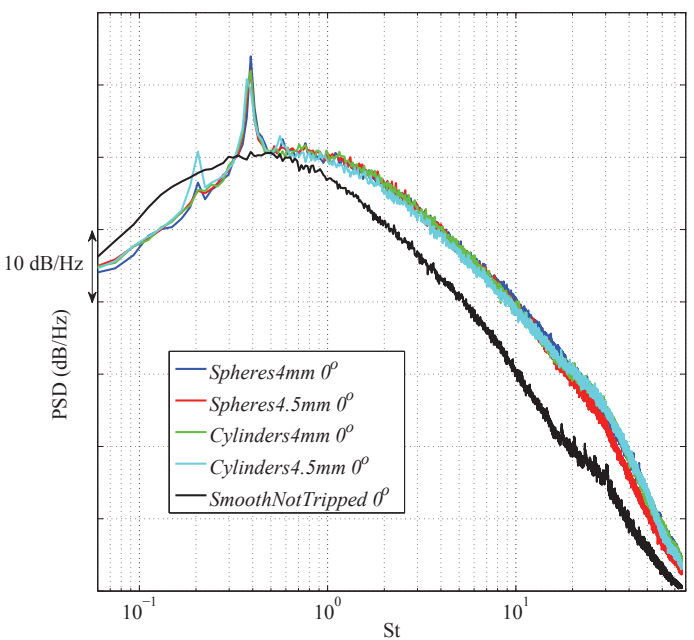

(a) $0^{\circ}$

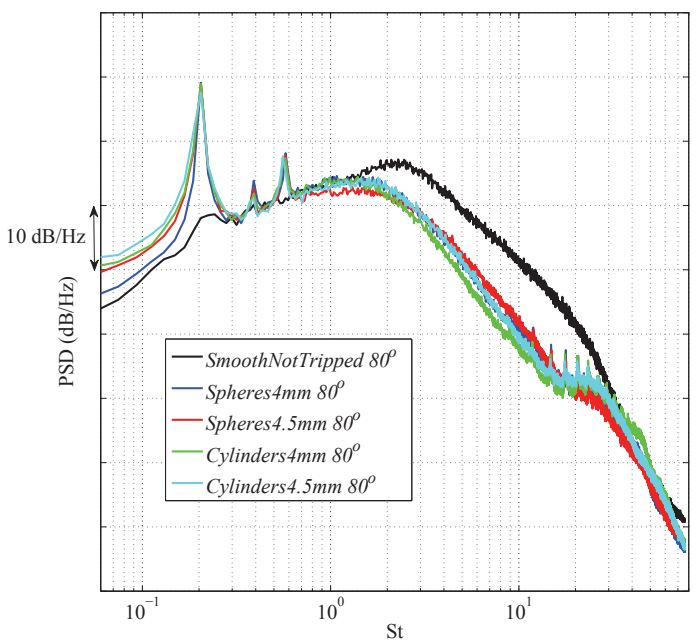

(c) $80^{\circ}$

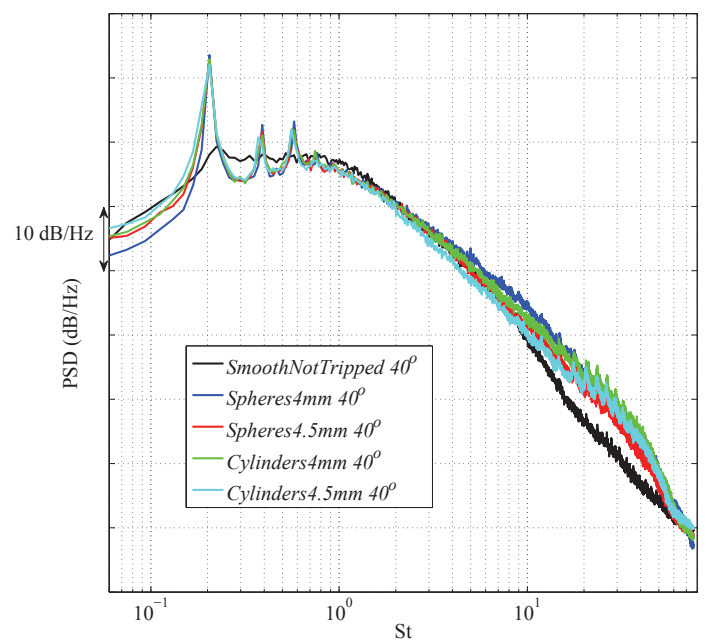

(b) $40^{\circ}$

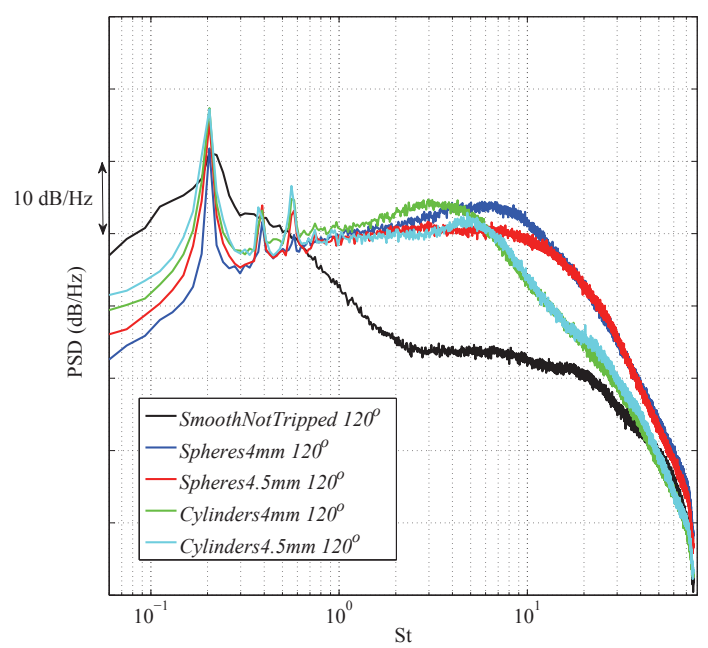

(d) $120^{\circ}$

Figure 14. Measured on-surface pressure spectra of the rough circular cylinders at $R e=3.2 \cdot 10^{5}$ (angle measured from downstream stagnation line). 


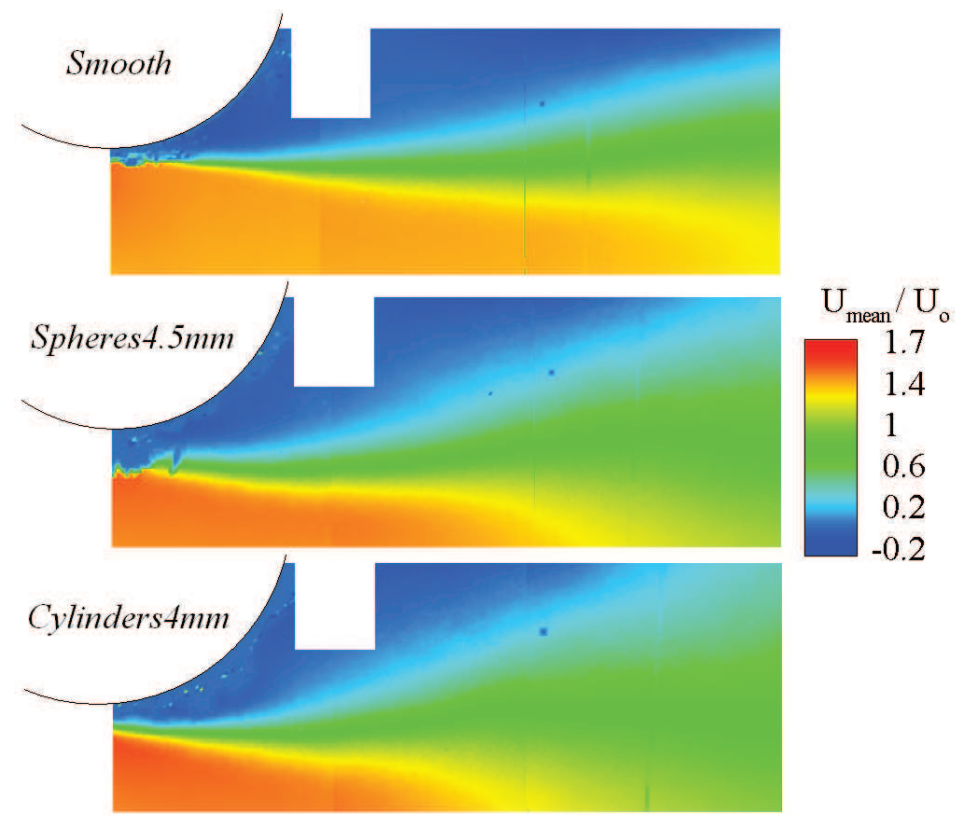

Figure 15. Mean x-velocity fields of the circular cylinders with distributed roughness, measured using PIV.

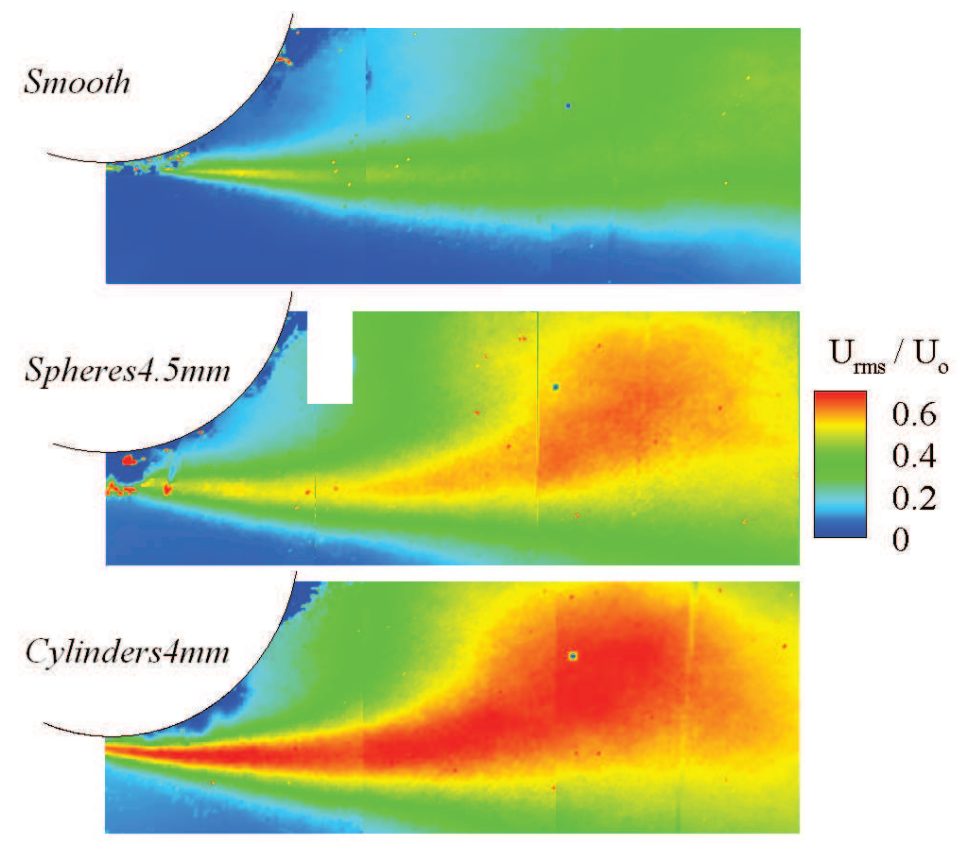

Figure 16. RMS x-velocity fields of the circular cylinder with distributed roughness, measured using PIV. 


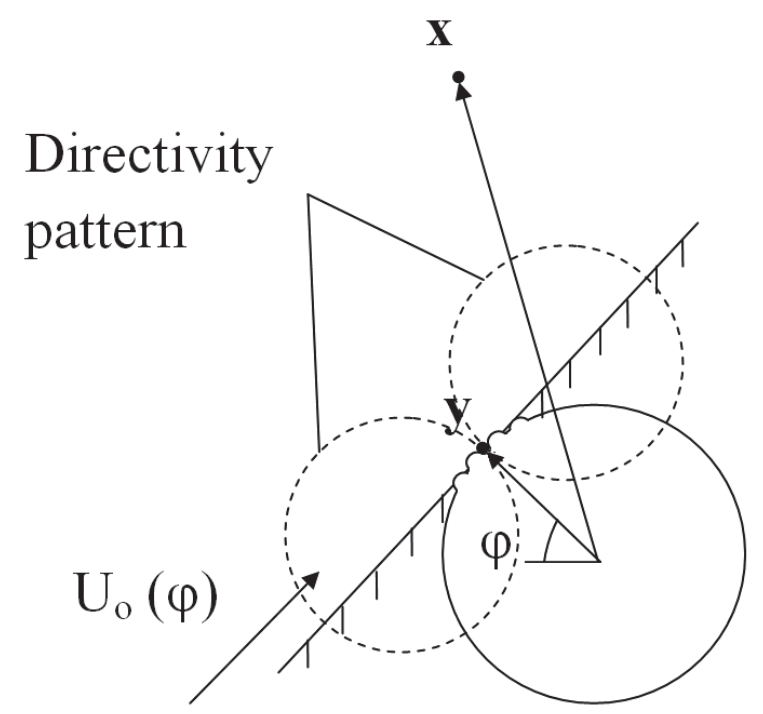

Figure 17. Flat geometry used in the circular cylinder prediction model instead of the real cylindrical geometry. 


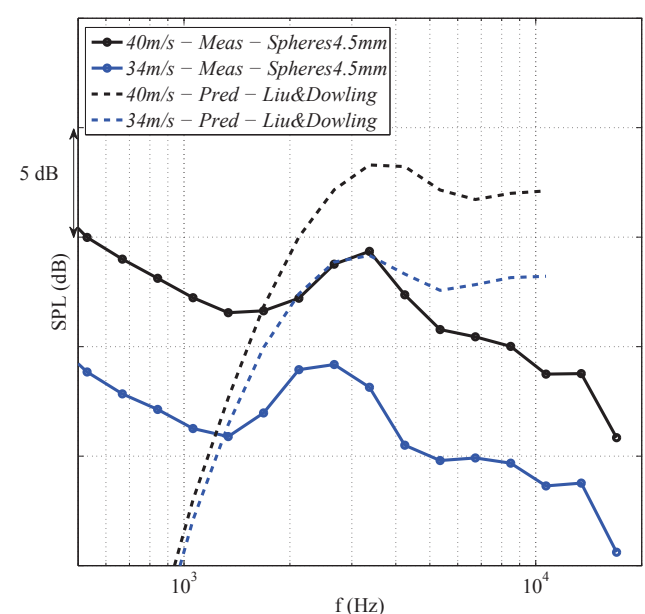

(a) $\theta=86^{\circ}$

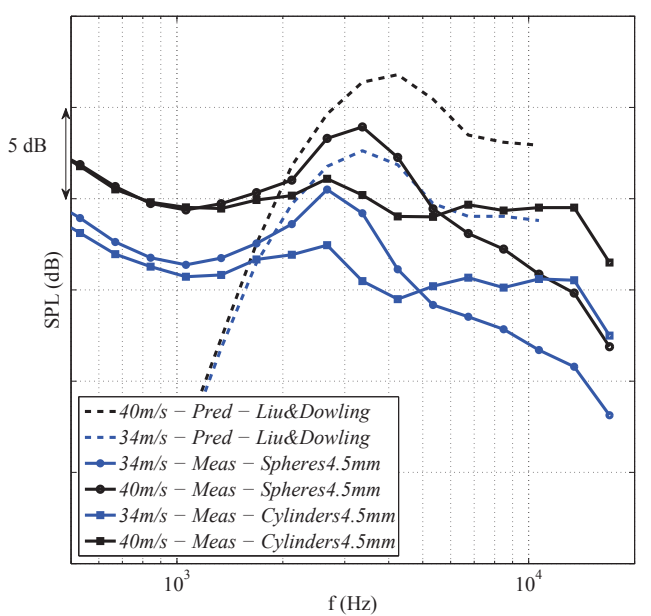

(b) $\theta=45^{\circ}$

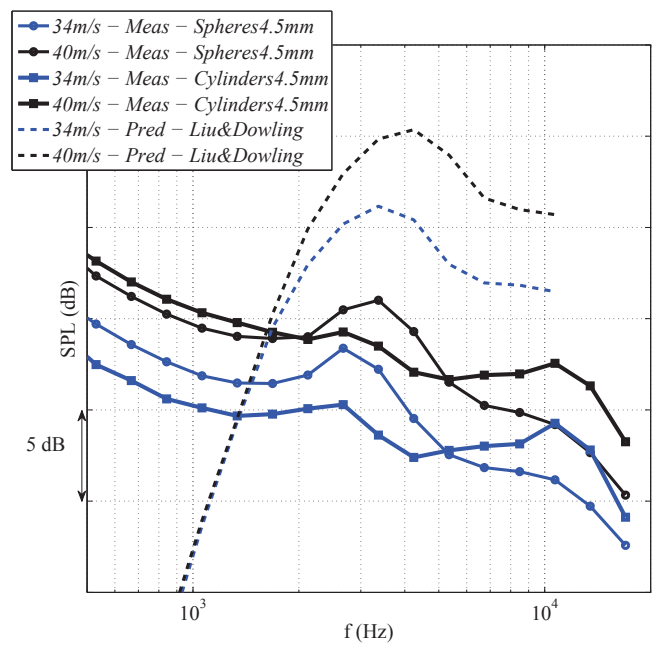

(c) $\theta=28^{\circ}$

Figure 18. Measured and predicted 1/3-octave band spectra of the far field noise for the circular cylinder. 